\title{
Correlative Imaging to Study Hemostats
}

\author{
J.P. DiOrio*, Y. Delmotte**, M. A. Murphy*, S. Strathmann*, L. Stojanovic*, A. Yardimci***, J. \\ McCoy***, Z. Yang***, A. Stover***, L. Campo***, L. Brannan***, J. Campbell***, S. \\ Donovan***
}

* Baxter Technology Resources, *** Baxter Bioscience, Round Lake, Illinois

** Baxter Global R \& D, Nivelles, Belgium

Baxter Healthcare Corporation currently has on the market wound management products which are used to control bleeding (Hemostasis). FloSeal [Hemostatic Matrix] is composed of a cross-linked gelatin matrix combined with thrombin to produce a highly viscous gel that functions as a topical hemostatic agent used to control bleeding when conventional surgical procedures are ineffective or impractical [1]. Tisseel [Fibrin Sealant] is a two component fibrin sealant [2] composed of sealer protein (mostly fibrinogen) and thrombin. It is indicated for use as an adjunct to hemostasis in surgeries involving cardiopulmonary bypass and splenic injury when control of bleeding by conventional surgical techniques is ineffective or impractical. Correlative microscopic techniques were used to study these products and as well as to develop methods to investigate the interactions of the biologics with the matrix material. A sintered polyethylene material was also examined that is part of a novel in-line device in development for mixing of the above biologics.

Sintered polyethylene disc material was examined with micro computerized (axial) tomography (Micro-CT: Skyscan 1172). Projection images were subsequently reconstructed to generate slices (cross sections). Palladium coated material was also examined in the scanning electron microscope (SEM: JEOL 6300F at $5 \mathrm{kV}$ ). Quantum Dot labeled fibrinogen (Q-dot ${ }^{\circledR}$;Invitrogen) was examined in the transmission electron microscope (TEM: JEOL 1400 at $120 \mathrm{kV}$ ) on carbon coated grids either alone or contrasted with $2 \%$ Uranyl Acetate. Tisseel VHSD sealer protein spiked with the Q-Dot labeled fibrinogen (1:50) was clotted with diluted thrombin. The samples were fixed in $2 \%$ glutaraldehyde and for SEM were dehydrated, air-dried out of hexamethyldisilazane, coated with palladium and imaged on the above SEM. Fixed TEM samples were osmicated followed by dehydration and embedment in Spurr's resin. Thin sections were stained with uranyl acetate and lead citrate and were imaged on the above TEM. In order to mimic a FloSeal clot formed in the presence of plasma, diluted Tisseel sealer protein was added to FloSeal gelatin granules and thrombin. It was then fixed in $2 \%$ glutaraldehyde and processed for TEM as described. For laser scanning confocal microscopy (Nikon A1RSi: $488 \mathrm{~nm}, 561 \mathrm{~nm}$ excitation), diluted Tisseel sealer protein was spiked with $0.1 \%$ Alexa Fluor fibrinogen 546 (Invitrogen), added to FloSeal and allowed to clot.

The sintered polyethylene material is seen in Figures 1 and 2 by Micro-CT and SEM, respectively. The morphology is similar by both techniques. In Figure 3, the Q-Dot labeled fibrinogen is seen in the TEM negatively stained, which reveals the electron dense core as well as the outside functional groups, which are not seen without the stain (inset). Fibrin formed in the presence of the Q-Dots is shown by SEM in Figure 4 as well as in TEM (inset) showing the individual Q-dots in a thin section. FloSeal granules with added fibrinogen are shown in Figures 5 and 6 by TEM and confocal microscopy, respectively. A similar morphology is seen with fibrin formation associated with the gelatin matrix. In Figure 6, the gelatin particle is visible due to auto-fluorescence. 


\section{References}

[1] C. Mehmet, et al., J. Card. Surg. 18 (2003) 486 and Ann. Thorac. Surg. 69 (2000) 1376.

[2] S. Helgerson et al., Encyclo. Biomat. Biomed Engin. (2004) 603.
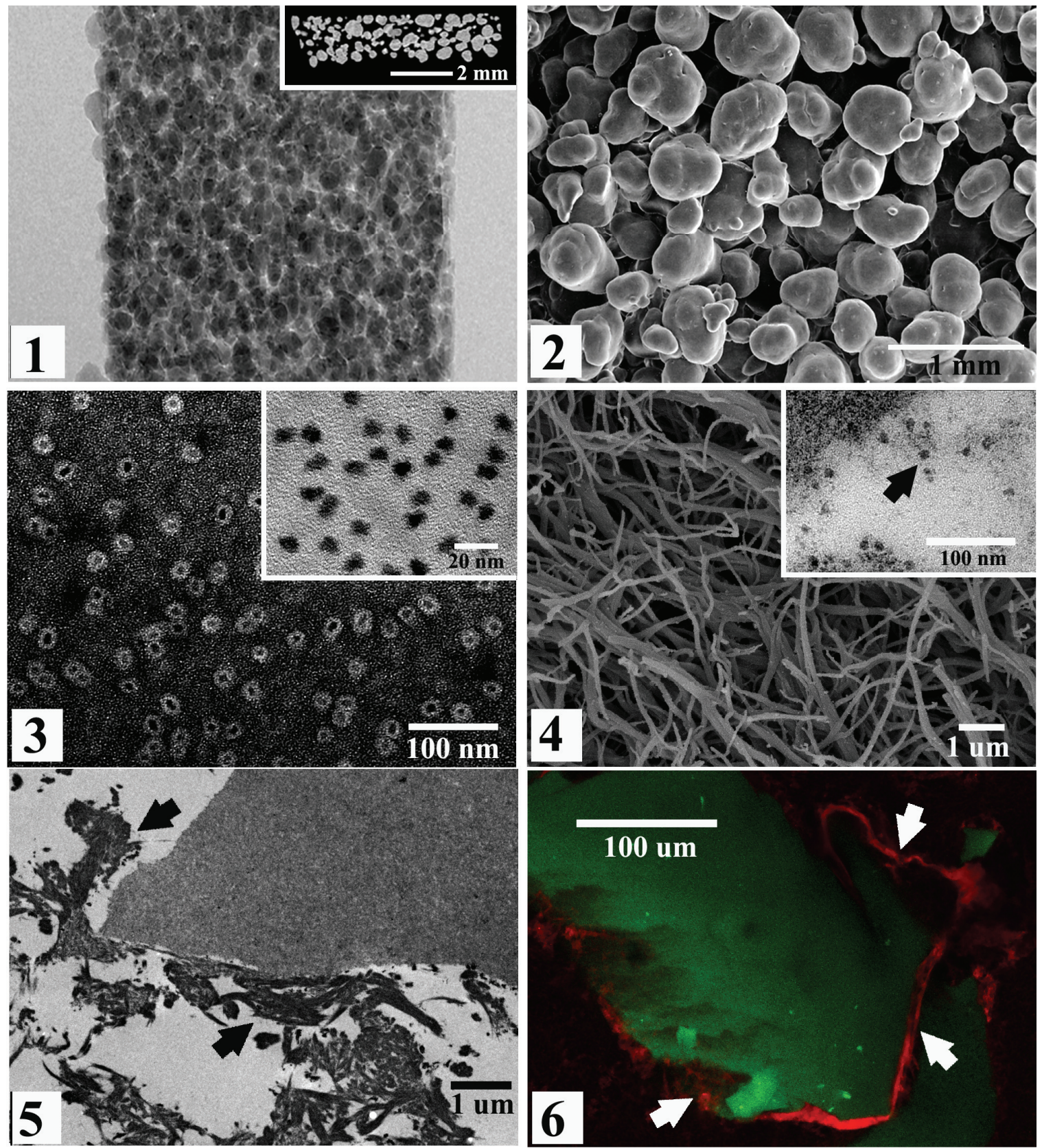

Fig. 1. Micro-CT projection image of sintered polyethylene material. Inset: horizontal cross section. Fig. 2. SEM of sintered polyethylene material.

Fig. 3. TEM of negatively stained fibrinogen labeled Q-Dots. Inset: no stain.

Fig. 4. SEM of fibrin with Q-Dot labeled fibrinogen. Inset: TEM section, arrow at sectioned Q-Dot. Fig. 5. TEM of fibrin (arrows) and FloSeal gelatin granule.

Fig. 6. Confocal image of granule and fibrin (arrows) formed in presence of Alexa fibrinogen. 